\title{
NON-LTE SPECTRA OF IRON GROUP ELEMENTS FOR CSPN
}

\author{
S.R. BECKER and K. BUTLER \\ Universitätssternwarte München, Scheinerstraße 1, 8000 München 80, Germany
}

\section{The calculations}

UV spectra of CSPN are dominated by lines from ions of iron group elements. Recently we have developed a non-LTE model of the FeV spectrum putting particular emphasis on this spectral region (Becker and Butler, 1992a). Lines of NiIV and Niv are major contributors to blends with the dominating Fev lines. Meanwhile, non-LTE models for $\mathrm{Ni} I \mathrm{~V}, \mathrm{Niv}$ and Nivi have been developed (Becker and Butler, 1992b) and the non-LTE model spectra composed of lines from all four ions can be compared to the observations.

In this paper we modelled the spectra of two CSPN (IC3568, NGC6826). IUE high resolution spectra were available and stellar parameters were taken from Mendez el al., 1990. The presence of a stellar wind has been accounted for according to the analytical stellar wind model by Kudritzki (1992a).

A resulting theoretical spectrum is shown in fig. 1 together with the observations. It is obvious from this that the major limitation for determination of abundances is currently given by the quality of the observations. HST spectra, when available, allow a reliable determination of the iron abundance (see Becker and Butler, 1992a, Kudritzki, 1992b).

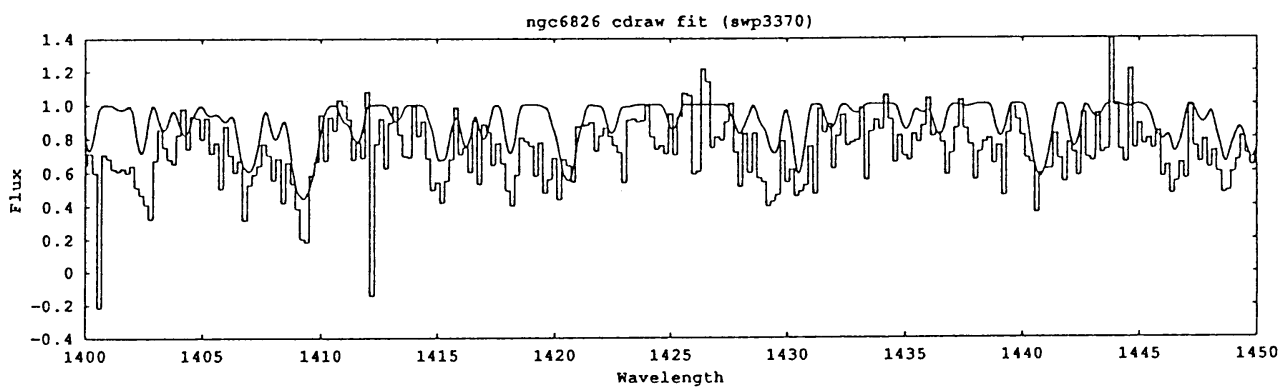

Figure 1: Comparison of a theoretical non-LTE spectrum with an IUE observation of NGC 6826 .

\section{REFERENCES}

Becker, S.R. and Butler, K., 1992a, A\&A, in press.

Becker, S.R. and Butler, K., 1992b, A\&A, in preparation.

Kudritzki, R.P., 1992a, A\&A in press.

Kudritzki, R.P., 1992b, this book.

Mendez, R.H., Herrero, A. and Manchado, A., 1990, A\&A, 229, 152. 